\title{
A GEOMETRIC SUFFICIENT CONDITION FOR EXISTENCE OF STABLE TRANSITION LAYERS FOR SOME REACTION-DIFFUSION EQUATIONS
}

\author{
Arnaldo Simal do Nascimento Janete Crema *
}

\begin{abstract}
Given a finite number of suitably distributed hypersurfaces in a bounded domain, we show how the spatial heterogeneities of a reaction-diffusion problem should depend on the geometry of the hypersurfaces in order to give rise to a one-parameter family of layered stable stationary solutions. These solutions in turn develop internal transition layers and have each hypersurface as an interface separating the regions where the solution is close to different constant equilibria.
\end{abstract}

\section{Introduction}

The main concern in this paper is the issue of how spatial heterogeneities in some reaction-diffusion equations can give rise to stable spatially heterogeneous stationary solutions.

Specifically we will consider diffusion processes governed by the following evolution problem:

$$
\left.\begin{array}{l}
\frac{\partial v_{\varepsilon}}{\partial t}=\varepsilon^{2} \operatorname{div}\left(a(x) \nabla v_{\varepsilon}\right)+f\left(x, v_{\varepsilon}\right), \quad(t, x) \in \mathbb{R}^{+} \times \Omega \\
v_{\varepsilon}(0, x)=\phi(x), \quad x \in \Omega \\
\frac{\partial v_{\varepsilon}}{\partial \hat{n}}=0, \quad(t, x) \in \mathbb{R}^{+} \times \partial \Omega
\end{array}\right\}
$$

where $\varepsilon$ is a small positive parameter, $\Omega \subset \mathbb{R}^{N}(N \geq 2)$ is a smooth bounded domain, $\hat{n}$ the inward normal to $\partial \Omega$ and $a$ is a strictly positive function in $C^{2}(\bar{\Omega})$.

*The first author was supported by CNPq. Key words. reaction-diffusion system, internal transition layer, equal-area condition. AMS subject classifications. 35B25, 35B35, 35K57, 35R35. 
Given an arbitrary smooth hypersurface $S \subset \Omega$ without boundary, we establish sufficient conditions on the difusibility function $a(x)$, the reaction term $f(x, \cdot)$ and the geometry of $S$ so that (1.1) possesses a stable stationary solution which develops internal transition layer, as $\varepsilon \rightarrow 0$, with interface $S$.

The reaction term $f \in C^{1}(\Omega \times \mathbb{R})$ is required to satisfy the following hypotheses:

$\left(f_{1}\right)$ There exist constants $\alpha$ and $\beta$ and a function $\theta \in C(\bar{\Omega})$ satisfying $\alpha<$ $\theta(x)<\beta, f(x, \alpha)=f(x, \beta)=f(x, \theta(x)) \equiv 0$ and $f_{v}(x, \alpha)<0, f_{v}(x, \beta)<$ $0, \forall x \in \bar{\Omega}$.

$\left(f_{2}\right) \int_{\alpha}^{\beta} f(x, \xi) d \xi=0$ (the equal-area condition) and $\int_{\alpha}^{v} f(x, \xi) d \xi<0, \quad \forall v \in(\alpha, \beta), \quad \forall x \in \Omega$.

$\left(f_{3}\right)$ There exist constants $a, b$ e $\sigma$ such that $|f(x, u)| \leq a+b|u|^{\sigma}$ with $1 \leq \sigma<$ $\frac{N+2}{N-2}$, if $N \geq 3$ and $1 \leq \sigma<\infty$ if $N=2$.

In order to put our work into perspective we set

$$
F(x, v) \stackrel{\text { def }}{=}-\int_{\alpha}^{v} f(x, \xi) d \xi
$$

which, by virtue of $\left(f_{2}\right)$, is positive in $(\alpha, \beta)$ for $\forall x \in \Omega$ and define the positive function

$$
h(x) \stackrel{\text { def }}{=} a^{1 / 2}(x) \int_{\alpha}^{\beta} F^{1 / 2}(x, \xi) d \xi .
$$

Also let $S \subset \Omega$ be a smooth compact hypersurface without boundary with principal curvatures at $y \in S$ denoted by $\kappa_{i}(y)(i=1, \ldots, N-1)$, mean curvature by $H(y)$ and the inward normal vector field to $S$ by $\nu$. Given a point $y \in S$, written in the local representation of $S$ as $y=\left(y^{\prime}, \varphi\left(y^{\prime}\right)\right)$ for some smooth function $\varphi, y^{\prime} \in \mathbb{R}^{\mathbb{N}-\nVdash}$ and small $\delta$ we set

$$
\Lambda\left(y^{\prime}, \delta\right) \stackrel{\text { def }}{=} h(y+\delta \nu(y)) \prod_{i=1}^{N-1}\left(1-\delta \kappa_{i}(y)\right) .
$$

Suppose that for each $y \in S$ fixed, $\delta=0$ is a strict local minimum of $\Lambda$. 
Then we prove that (1.1) possesses a family of stable stationary solutions $\left\{v_{\varepsilon}\right\}$ which develops internal transition layers, as $\varepsilon \rightarrow 0$, with interface $S$ separating $\Omega$ in two regions $\Omega_{\alpha}$ and $\Omega_{\beta}$. Moreover $v_{\varepsilon}$ converges uniformly in compact sets of $\Omega_{\alpha}\left(\Omega_{\beta}\right)$ to $\alpha(\beta)$. A similar result is easily obtained for a finite number of nested hypersurfaces and in this case the solution will be referred to as a multiple-layer pattern.

The assumption that $\delta=0$ is a strict local minimum of $\Lambda$ has a geometric meaning. Indeed by setting, for each $y=\left(y^{\prime}, \varphi\left(y^{\prime}\right)\right) \in S$,

$$
\lambda(\delta) \stackrel{\text { def }}{=} h(y+\delta \nu(y))
$$

a straightforward computation shows that a sufficient condition for this hypothesis to hold is

$$
\begin{aligned}
\lambda^{\prime}(0) & =2 h(y) H(y) \\
\lambda^{\prime \prime}(0) & >h(y)\left\{\sum_{i=1}^{N-1} \kappa_{i}^{2}(y)+(N-1)^{2} H^{2}(y)\right\}
\end{aligned}
$$

Conditions (1.2) and (1.3) are simpler in two-dimensional domains. Indeed suppose $N=2$ and $S=\gamma(s)$ is a smooth simple closed curve in $\Omega$ whose signed curvature is denoted by $\kappa(s), 0 \leq s \leq L, L$ being its total arc-length. Then (1.2) and (1.3) become

$$
\begin{gathered}
\lambda^{\prime}(0)=h(\gamma(s)) \kappa(s), \quad \forall s \in[0, L) \\
\lambda^{\prime \prime}(0)>2 h(\gamma(s)) \kappa^{2}(s), \quad \forall s \in[0, L)
\end{gathered}
$$

In this case (1.4) and (1.5) conform with the conditions obtained in [1] and [2], where $S=\gamma(s)$ was assumed to be a level-curve of $h$. This hypothesis has been dropped here.

Note that as $s$ increases the slope of $\lambda(\delta)$ at $\delta=0$ will change sign whenever $\kappa(s)$ does, while its positive concavity increases with the curvature of $\gamma$. Also at those points $\gamma(s)$ where $\kappa(s)=0$ (saddle or planar points), $\lambda(\delta)$ will have a local strict minimum at $\delta=0$. 
We know from [4] that the equal-area condition in $\left(f_{2}\right)$ is a necessary hypothesis for the existence of a family of stationary solutions to (1.1) developing internal transition layer as will be the case here.

Note that it is not required that $\alpha, \theta(x)$ and $\beta$ be consecutive zeros of $f(x, \cdot)$. Indeed there may be other zeros of $f(x, \cdot)$ lying between $\alpha$ and $\beta$ for which the equal-area condition may hold. However it is required through the second assumption in $\left(f_{2}\right)$ that $F(x, v)$ satisfies $F(x, \alpha)=F(x, \beta)=0$ and $F(x, v)>0$ for $v \in(\alpha, \beta)$, in other words, $F(x, \cdot)$ is a double-well potential in $[\alpha, \beta]$.

As for hypothesis $\left(f_{3}\right)$ it is required only because for a technical reason we need the corresponding energy functional to be $C^{1}$.

Throughout this work we will refer to spatially heterogeneous stable stationary solutions to (1.1) as patterns, for short.

For scalar equations like the one being considered, patterns can be created either through nonconvexity of the domain or spatial heterogeneities.

The question of how spatial heterogeneities can give rise to patterns has been subject of research for quite some time.

For the one-dimensional case when $f(x, v)=f(v)$ is close to a piece-wise constant function the existence of pattern induced by the function $a$ was considered in [6] and [7].

In [3] this question was addressed for the case in which $\Omega$ is the unit $N$ dimensional ball, $a$ a radially symmetric function and $f(x, v)=f(v)$.

The existence of multiple-layer pattern for (1.1) was also considered in [11] for the case $\Omega=[0,1], \alpha=0, \beta=1$ and the reaction term takes the form $v(1-v)\left[v-\left(1 / 2+a_{1} \varepsilon+o\left(\varepsilon^{2}\right)\right)\right]$ with $a_{1}=$ constant.

The results of [11] was generalized in [2] to the case in which $\Omega$ is a smooth arbitrary two-dimensional domain, $f(x, v ; \varepsilon)=h_{2}(x) v(1-v)(v-\gamma(x, \varepsilon))$, where $\gamma(x, \varepsilon)=1 / 2+g_{\varepsilon}(x)$ with $g_{\varepsilon}(x)=o(\varepsilon)$ as $\varepsilon \rightarrow 0$, uniformly in $\Omega$ and the nested curves $\left.S_{i}(i=1, \ldots, m)\right)$ were assumed to be level curves of the function $\left(a h_{2}\right)^{1 / 2}$.

Although we do not care here, the reaction term $f$ might have been allowed 
to depend on $\varepsilon$ in an appropriate manner so as to generalize the results of [2].

Our approach gives existence, stability and the geometrical structure of patterns for (1.1) simultaneously and the method of proof is based on $\Gamma$-convergence.

\section{Preliminaries on $B V$-functions}

Before proving the main result we recall some notation on measures and results on functions of bounded variation. The reader is referred to [12] for further background. The Lebesgue measure in $\mathbb{R}^{N}$ is denoted by $\mathcal{L}$ and the $m$-dimensional Hausdorff measure by $\mathcal{H}^{m}$.

If $\mu$ is a Borel measure on $\Omega$ with values in $\left[0,+\infty\left[\right.\right.$ or in $\mathbb{R}^{N}, N \geq 1$, its total variations is denoted by $|\mu|$ and the integral of a $|\mu|$-integrable function $f$ will be denoted by $\int_{\Omega} f|\mu|$. The space $B V(\Omega)$ of functions of bounded variation in $\Omega$ is defined as the set of all functions $v \in L^{1}(\Omega)$ whose distributional gradient $D v$ is a Radon measure with bounded total variation in $\Omega$, i.e.,

$$
|D v|(\Omega)=\sup _{\substack{\sigma \in C_{0}^{1}\left(\Omega, \mathbb{R}^{N}\right) \\|\sigma| \leq 1}} \int_{\Omega} v(x) \operatorname{div} \sigma(x) d \mathcal{L}<\infty .
$$

If $v \in W_{\text {loc }}^{1,1}$ then the total variation measure satisfies $|D v|=\mathcal{L}\llcorner|\nabla v|$, i.e., $|D v|=|\nabla v| d \mathcal{L}$, where $\nabla$ denotes the usual gradient.

We denote by $B V(\Omega ;\{\alpha, \beta\})$ the class of all $u \in B V(\Omega)$ which take values $\alpha, \beta$ only.

The essential boundary of a set $E \subset \mathbb{R}^{N}$ is the set $\partial_{*} E$ of all points in $\Omega$ where $E$ has neither density 1 nor density 0 . If a set $E \subset \Omega$ has finite perimeter in $\Omega$ then $\partial_{*} E$ is rectifiable, and we may endow it with a measure theoretic normal $\nu_{E}$ so that the measure derivative $D \chi_{E}$ is represented as

$$
D \chi_{E}(B)=\int_{B \cap \partial_{*} E} \nu_{E} d \mathcal{H}^{N-1}
$$

for every Borel set $B \subset \Omega$. A Borel set $B \subset \mathbb{R}^{n}$ has finite perimeter in the open set $\Omega$ if

$$
\operatorname{Per}_{\Omega}(B):=\left|D \chi_{B}\right|(\Omega)<\infty,
$$


where $\chi_{B}$ is the characteristic function of $B$.

The following version of the coarea formula will be often used. Let $v \in$ $B V(\Omega)$ and suppose that $f$ is a continuous function in $\Omega$. Then there holds

$$
\int_{\Omega} f|D v|=\int_{\infty}^{\infty}\left\{\int_{\Omega \cap \partial_{*}\{v>\xi\}} f d \mathcal{H}^{N-1}\right\} d \xi
$$

Definition 2.1 A family $\left\{E^{\varepsilon}\right\}_{0<\varepsilon \leq \varepsilon_{0}}$ of real-extended functionals defined in $L^{1}(\Omega)$ is said to $\Gamma$-lower converge, as $\varepsilon \rightarrow 0$, to a functional $E^{0}$, and we write

$$
\Gamma\left(L^{1}(\Omega)^{-}\right)-\lim _{\varepsilon \rightarrow 0} E^{\varepsilon}(v)=E^{0}(v)
$$

if:

- For each $v \in L^{1}(\Omega)$ and for any sequence $\left\{v_{\varepsilon}\right\}$ in $L^{1}(\Omega)$ such that $v_{\varepsilon} \rightarrow v$ in $L^{1}(\Omega)$, as $\varepsilon \rightarrow 0$, there holds

$$
E^{0}(v) \leq \lim _{\varepsilon \rightarrow 0} \inf _{\varepsilon} E^{\varepsilon}\left(v_{\varepsilon}\right)
$$

- For each $v \in L^{1}(\Omega)$ there is a sequence $\left\{w_{\varepsilon}\right\}$ in $L^{1}(\Omega)$ such that $w_{\varepsilon} \rightarrow v$ in $L^{1}(\Omega)$, as $\varepsilon \rightarrow 0$ and also $E^{0}(v) \geq \lim \sup _{\varepsilon \rightarrow 0} E^{\varepsilon}\left(w_{\varepsilon}\right)$.

Definition 2.2 We say that $v_{0} \in L^{1}(\Omega)$ is an $L^{1}$-local minimiser of $E^{0}$ if there is $\rho>0$ such that

$$
E^{0}\left(v_{0}\right) \leq E^{0}(v) \text { whenever } 0<\left\|v-v_{0}\right\|_{L^{1}(\Omega)}<\rho
$$

Moreover if $E^{0}\left(v_{0}\right)<E^{0}(v)$ for $0<\left\|v-v_{0}\right\|_{L^{1}(\Omega)}<\rho$, then $v_{0}$ is called an isolated $L^{1}$-local minimiser of $E^{0}$.

The following theorem in its abstract version is due to De Giorgi and version we need here can be found in [9].

Theorem 2.3 Suppose that a family of real-extended functionals $\left\{E^{\varepsilon}\right\}, \Gamma$-lower converges, as $\varepsilon \rightarrow 0$, to a real-extended functional $E^{0}$ and the following hypotheses are satisfied: 
(2.3.i) Any sequence $\left\{u_{\varepsilon}\right\}_{\varepsilon>0}$ such that $E^{\varepsilon}\left(u_{\varepsilon}\right) \leq$ constant $<\infty$, is compact in $L^{1}(\Omega)$.

(2.3.ii) There exists an isolated $L^{1}$-local minimiser $v_{0}$ of $E^{0}$.

Then there exists $\varepsilon_{0}>0$ and a family $\left\{v_{\varepsilon}\right\}_{0<\varepsilon \leq \varepsilon_{0}}$ such that $v_{\varepsilon}$ is an $L_{1}$-local minimiser of $E^{\varepsilon}$ and $\left\|v_{\varepsilon}-v_{0}\right\|_{L^{1}(\Omega)} \rightarrow 0$, as $\varepsilon \rightarrow 0$.

What follows has to do with the local representation of a smooth hypersurface $S$. Trying to reach a broader readership we decide not to use the language of local charts and atlas for compact imbedded submanifolds of $\mathbb{R}^{N}$ and present instead a self-contained argument.

Given a $C^{2}$ hypersurface $S$ a change of coordinates $\Xi$ can be defined which straightens portions of $S$. Indeed if $y \in S$ then by a rotation and a translation of coordinates we may assume that $y$ is the origin of the system and that the internal normal $\nu(y)$ to $S$ at $y$ lies in the direction of the positive $x_{N}$-coordinate axis.

Let $T_{S}(y)$ denote the hyperplane tangent to $S$ at $y$. Then there is a neighborhood $\mathcal{V}(y)$ of $y$ in $\mathbb{R}^{N}$ and a function $\varphi \in C^{2}\left(\mathcal{V} \cap T_{S}(y)\right)$ such that by setting $y^{\prime}=\left(y_{1}, \ldots, y_{N-1}\right)$ then

$$
S \cap \mathcal{V}(y)=\left\{\left(y^{\prime}, y_{N}\right), y_{N}=\varphi\left(y^{\prime}\right)\right\}
$$

Since $S$ is compact, there are finitely many points, say, $y_{k} \in S(k=1, \ldots, K)$ such that $\cup_{k=1}^{K}\left(S \cap \mathcal{V}\left(y_{k}\right)\right)$ is a covering of $S$. Of course, for each $y_{k}$ there will be a function $\varphi_{k}$ for which the above local representation of $S$ holds. However for simplicity we will drop the subindex $k$, thus writing only $\varphi$, regardless of the neighborhood $S \cap \mathcal{V}\left(y_{k}\right)$ it represents.

Let $\delta(x)=\operatorname{dist}(x, S)$ stand for the usual signed distance function which is positive inside the open region enclosed by $S$ and negative outside that region. Also define a tubular neighborhood of $S$ by

$$
N_{\delta_{0}}(S)=\left\{x \in \Omega:|\delta(x)|<\delta_{0}\right\}
$$


where $\delta_{0}$ is taken small enough so that $\delta \in C^{2}\left(N_{\delta_{0}}\right)$. For each $x \in N_{\delta_{0}}$ there exits a unique point $y=y(x) \in S$ such that $|y-x|=\delta(x)$.

These two points are related by $x=y+\nu(y) \delta(x)$. For any $\delta$ such that $0<\delta \leq \delta_{0}$ we set

$$
I_{\delta} \stackrel{\text { def }}{=}(-\delta, \delta)
$$

For a fixed $y_{k} \in S, k \in\{1, \ldots, K\}$, define a mapping

$$
\Xi:\left(T_{S}\left(y_{k}\right) \cap \mathcal{V}\left(y_{k}\right)\right) \times I_{\delta_{0}} \longrightarrow \mathbb{R}^{N}
$$

by $\Xi\left(y^{\prime}, \delta\right)=\left(y^{\prime}, \varphi\left(y^{\prime}\right)\right)+\delta \nu\left(y^{\prime}, \varphi\left(y^{\prime}\right)\right)$.

The orthogonal projection of $S \cap \mathcal{V}\left(y_{k}\right)$ onto $T_{S}\left(y_{k}\right)$ will be denoted by $\widetilde{T}_{S}\left(y_{k}\right)$ and in general is strictly contained in $T_{S}\left(y_{k}\right) \cap \mathcal{V}\left(y_{k}\right)$. By setting

$$
\Xi\left(\widetilde{T}_{S}\left(y_{k}\right) \times I_{\delta_{0}}\right)=C\left(y_{k}\right)
$$

then $\Xi$ defines a diffeomorphism between $\widetilde{T}_{S}\left(y_{k}\right) \times I_{\delta_{0}}$ and $C\left(y_{k}\right)=\left(y^{\prime}, \varphi\left(y^{\prime}\right)\right)+$ $\nu(y) \delta$, for $y^{\prime} \in \widetilde{T}_{S}\left(y_{k}\right)$ and $\delta \in I_{\delta_{0}}$.

Clearly by taking $\delta_{0}$ smaller if necessary we have $N_{\delta_{0}}(S) \subset \bigcup_{k=1}^{K} C\left(y_{k}\right)$.

The underlying theorem used in our approach will depend on a suitable disjoint covering of $N_{\delta_{0}}$ which, for the sake of brevity we rather define at this point and work with it from now on.

So let us define a new mutually disjoint family of sets by putting $C_{k}=$ $C\left(y_{k}\right)(k=1, \ldots, K)$ and

$$
W_{1}=C_{1} \text { and } W_{k}=C_{k} \backslash\left\{\cup_{j=1}^{k-1} C_{j}\right\} \quad(k=2, \ldots, K)
$$

It follows that $W_{i} \cap W_{j}$ is empty for $i \neq j$ and $N_{\delta_{0}}(S)=\cup_{k=1}^{K} W_{k}$.

Let

$$
\widetilde{W}_{k} \stackrel{\text { def }}{=} \Xi^{-1}\left(W_{k}\right)=M_{k} \times I_{\delta_{0}}
$$

where $M_{k} \subset \widetilde{T}_{S}\left(y_{k}\right)$ is such that $\partial M_{k}$ is $\mathcal{H}^{N-2}$-a.e. smooth.

Unless otherwise said, we use "tilde" to denote a function, a set, a measure, etc., in the new coordinates. 
If $\kappa_{1}, \ldots, \kappa_{N-1}$ denote the principal curvatures of $S$ at $\left(y^{\prime}, \varphi\left(y^{\prime}\right)\right)$ and $\Xi\left(y^{\prime}, \delta\right)=$ $x$, then the Jacobian matrix of $\Xi$ is given by

$$
(D \Xi)(y \prime, \delta)=\operatorname{diag}\left[1-\kappa_{1} \delta, \ldots, 1-\kappa_{N-1} \delta, 1\right]
$$

and its determinant by

$$
J_{\Xi}(y \prime, \delta)=\prod_{i=1}^{N-1}\left(1-\kappa_{i}\left(y^{\prime}, \varphi\left(y^{\prime}\right)\right) \delta\right)
$$

for $(y \prime, \delta) \in \widetilde{W}_{k}, k=1, \ldots, K$. For $\delta_{0}$ sufficiently small there holds that $J_{\Xi}>0$.

For $v \in B V(\Omega)$, we set from now on

$$
\mu=D v, \tilde{\mu}_{1}=\frac{\partial \tilde{v}}{\partial y_{1}}, \ldots, \tilde{\mu}_{N-1}=\frac{\partial \tilde{v}}{\partial y_{N-1}} \text { and } \tilde{\mu}_{N}=J_{\Xi} \frac{\partial \tilde{v}}{\partial \delta} .
$$

The next lemma plays an important role in the proof of the main result and the reader is referred to [2] for the proof.

Lemma 2.4 With this notation let $v \in B V\left(W_{k}\right),(k=1, \ldots, K)$. Then, for any Borel set $B \subset W_{k}$, we have

$$
|\mu|(B) \geq\left(\sum_{i=1}^{N}\left(\left|\widetilde{\mu}_{i}\right|(\widetilde{B})\right)^{2}\right)^{1 / 2} .
$$

\section{Main Result}

For the sake of simplicity we first state our main result for just one hypersurface and afterwards treat the general case.

Let $S \subset \Omega$ be a compact smooth hypersurface without boundary that partition $\Omega$ in two open sets $\Omega_{\alpha}$ and $\Omega_{\beta}$. We may suppose that $\partial \Omega_{\alpha}=S$. Thus $\Omega=\Omega_{\alpha} \cup S \cup \Omega_{\beta}$.

For future reference we consider the following function

$$
v_{0}=\alpha \chi_{\Omega_{\alpha}}+\beta \chi_{\Omega_{\beta}}
$$

where $\chi_{A}$ stands for the characteristic function of the set $A$. 
Once $S$ is fixed we take a disjoint covering $W_{k}(k=1, \ldots, K)$ of $N_{\delta_{0}}(S)$ as above and recall that $\widetilde{W}_{k}=\Xi^{-1}\left(W_{k}\right)=M_{k} \times I_{\delta_{0}}$.

As mentioned before any function $g$ defined on $W_{k}$ is denoted in the new variable by $\tilde{g}=g \circ \Xi$.

Note that for $y \in S, y=\left(y^{\prime}, \varphi\left(y^{\prime}\right)\right)=\Xi\left(y^{\prime}, 0\right), J_{\Xi}\left(y^{\prime}, 0\right)=1$ and $\widetilde{g}\left(y^{\prime}, 0\right)=$ $g(y)$.

Recall that $N_{\delta_{0}}(S)=\cup_{k=1}^{K} W_{k}$ is the tubular neighborhood of $S$.

Theorem 3.1 Regarding (1.1), set

$$
h(x) \stackrel{\text { def }}{=} a^{1 / 2}(x) \int_{\alpha}^{\beta} F^{1 / 2}(x, \xi) d \xi, \quad x \in \Omega
$$

and consider the hypersurface $S$ with the notation as above. Let

$$
\Lambda\left(y^{\prime}, \delta\right) \stackrel{\text { def }}{=} \widetilde{h}\left(y^{\prime}, \delta\right) \prod_{i=1}^{N-1}\left(1-\kappa_{i}(y) \delta\right)
$$

and suppose that for each fixed $y^{\prime}, \delta=0$ is a strict local minimum of $\Lambda\left(y^{\prime}, \delta\right)$, i.e., for $\delta_{0}$ sufficiently small

$$
\Lambda\left(y^{\prime}, \delta\right)>\Lambda\left(y^{\prime}, 0\right)=\widetilde{h}\left(y^{\prime}, 0\right)=h(y), \delta \in I_{\delta_{0}}, \delta \neq 0
$$

Then for $\varepsilon_{0}$ small enough, there is a family $\left\{v_{\varepsilon}\right\}_{0<\varepsilon \leq \varepsilon_{0}}$ of stationary solutions of (1.1) such that for any $\varepsilon \in\left(0, \varepsilon_{0}\right)$ :

(3.1.i) $v_{\varepsilon} \in C^{2, \sigma}(\bar{\Omega}), 0<\sigma<1$ and $\alpha<v_{\varepsilon}(x)<\beta, \forall x \in \bar{\Omega}$.

(3.1.ii) $\left\|v_{\varepsilon}-v_{0}\right\|_{L^{1}(\Omega)} \stackrel{\varepsilon \rightarrow 0}{\longrightarrow} 0$, where $v_{0}$ is given by (3.1).

(3.1.iii) For $i \in\{\alpha, \beta\}$ and for any compact set $C \subset \Omega_{i}$ it holds that $v_{\varepsilon} \rightarrow i$ uniformly on $C$ as $\varepsilon \rightarrow 0$.

(3.1.iv) $v_{\varepsilon}$ is a stable stationary solution of (1.1).

Remark 3.2 As mentioned in the Introduction if (1.2) and (1.3) are assumed then (3.3) holds. 


\section{Proof of Theorem 3.1}

We start by setting up a suitable scenario in which the proof of Theorem 3.1 will be naturally cast into.

A stationary solution of (1.1) is a solution which satisfies the following boundary value problem:

$$
\left\{\begin{array}{l}
\varepsilon^{2} \operatorname{div}\left[a(x) \nabla v_{\varepsilon}\right]+f\left(x, v_{\varepsilon}\right)=0, \quad x \in \Omega \\
\nabla v_{\varepsilon}(x) \cdot \widehat{n}(x)=0, \quad \text { for } \quad x \in \partial \Omega
\end{array}\right.
$$

Next we define a family of functionals $E^{\varepsilon}: L^{1}(\Omega) \rightarrow \mathbb{R} \cup\{\infty\}$ by:

$$
E^{\varepsilon}(v)=\left\{\begin{array}{l}
\int_{\Omega}\left(\frac{\varepsilon a(x)}{2}|\nabla v|^{2}+\varepsilon^{-1} F(x, v)\right) d x, \text { if } v \in H^{1}(\Omega) \\
\infty, \text { otherwise. }
\end{array}\right.
$$

It is easy to see that any local minimiser $v_{\varepsilon}$ of $E^{\varepsilon}$ will be a weak solution to (4.1) and, by regularity, $v_{\varepsilon} \in C^{2, \nu}(\Omega), 0<\nu<1$.

At this point we truncate the functions $f$ in the following manner

$$
f_{c}(x, v)=\left\{\begin{array}{l}
f_{v}(x, \alpha)(v-\alpha), \text { for }-\infty \leq v<\alpha \\
f(x, v), \text { for } \alpha \leq v \leq \beta \\
f_{v}(x, \beta)(v-\beta), \text { for } \beta<v \leq \infty
\end{array}\right.
$$

We need this in order to conclude that the solutions been sought lie between $\alpha$ and $\beta$. By replacing $f$ with $f_{c}$ in $F$ we define $F_{c}$ and replacing $F$ with $F_{c}$ in $E^{\varepsilon}$ we accordingly define $E_{c}^{\varepsilon}$.

Remark that $\left|f_{c}(x, v)\right| \leq a|v|+b$, hence $F_{c}(x, \cdot)$ has quadratic growth and as such $E_{c}^{\varepsilon}$ is well defined and in fact it is a $C^{1}$ functional on $H^{1}(\Omega)$. Any critical point of $E_{c}^{\varepsilon}$ is a weak solution (in the $H^{1}$-sense) of the following boundary value problem

$$
\left\{\begin{array}{l}
\varepsilon^{2} \operatorname{div}\left[a(x) \nabla v_{\varepsilon}\right]+f_{c}\left(x, v_{\varepsilon}\right)=0, \quad x \in \Omega \\
\nabla v_{\varepsilon}(x) \cdot \widehat{n}(x)=0, \quad \text { for } \quad x \in \partial \Omega
\end{array}\right.
$$

It is our aim now to find a family of local minimisers of $E_{c}^{\varepsilon}$. 


\section{Theorem 4.1}

$$
\Gamma\left(L^{1}(\Omega)^{-}\right)-\lim _{\varepsilon \rightarrow 0} E_{c}^{\varepsilon}(v)=E_{c}^{0}(v)
$$

where

$$
E_{c}^{0}(v)=\left\{\begin{array}{l}
\int_{\Omega} h(x)\left|D \chi_{\{v=\beta\}}\right| \text { if } v \in B V(\Omega,\{\alpha, \beta\}) \\
\infty, \text { otherwise. }
\end{array}\right.
$$

Proof: In view of $\left(f_{1}\right)$ and $\left(f_{2}\right)$ and setting $\frac{\partial F_{c}}{\partial v}=F_{c, v}$ then

$$
\left.\begin{array}{l}
F_{c}(x, \alpha)=F_{c}(x, \beta)=0, \quad F_{c}(x, v) \in C^{2} \\
F_{c}(x, v)>0 \text { for any } v \in \mathbb{R}, v \neq \alpha, v \neq \beta \\
F_{c, v}(x, \alpha)=F_{c, v}(x, \beta)=0 \\
F_{c, v v}(x, \alpha)>0, F_{c, v v}(x, \beta)>0
\end{array}\right\}
$$

Now with these hypotheses, the proof of Theorem 2 [8], applies ipsis litteris.

Our goal now is to apply Theorem 2.3 to $E_{c}^{\varepsilon}$ and $E_{c}^{0}$. This will be accomplished by generalizing the argument given in [9] where the case $N=2, a \equiv 1$ and $f(x, v)=v-v^{3}$ was considered.

Since our next result is local in nature, given $S \subset \Omega$ a $C^{2}$-hypersurface we will use the same notation set forth in Section 3 with $\Omega$ replaced with $N_{\delta_{0}}(S)$ so that $N_{\delta_{0}}(S)=\Omega_{\alpha} \cup S \cup \Omega_{\beta}$.

Theorem 4.2 Suppose that (3.3) holds. Then with the notation above,

$$
v_{0}(x) \stackrel{\text { def }}{=} \alpha \chi_{\Omega_{\alpha}}(x)+\beta \chi_{\Omega_{\beta}}(x), \quad x \in N_{\delta_{0}}(S)
$$

is an $L^{1}$-local isolated minimiser of $E_{c}^{0}$ defined by (4.5).

Proof: Since the argument and the coordinates are local, a proof will be rendered first for any $W_{i}, i \in\{1, \ldots, K\}$, then for $N_{\delta_{0}}(S)=\cup_{i=1}^{K} W_{i}$. Hence throughout the proof we restrict $v_{0}$ to $W_{i}$ and keep the same notation.

It suffices to prove that, if $v \in B V\left(W_{i} ;\{\alpha, \beta\}\right)$ for $i \in\{1, \ldots, K\}$ and $0<\left\|v-v_{0}\right\|_{L^{1}\left(W_{i}\right)}<\rho_{i}$, for a suitable $\rho_{i}>0$, then $\int_{W_{i}} h|D v|>\int_{W_{i}} h\left|D v_{0}\right|$. 
We will say that $v$ is an admissible function in $W_{i}$ if $v \in B V\left(W_{i} ;\{\alpha, \beta\}\right)$ and $\left\|v-v_{0}\right\|_{L^{1}\left(W_{i}\right)}>0$ with $0<|\{v=\beta\}|<\left|W_{i}\right|$. Therefore for an admissible function $v$ we will often make use of the fact that $|D v|=\mathcal{H}^{N-1}\llcorner$ $\left(W_{i} \cap\left\{\partial_{*} v=\alpha\right\} \cap\left\{\partial_{*} v=\beta\right\}\right)$.

For future use, for any $\delta \in I_{\delta_{0}}$ we further denote a slice of $\widetilde{W}_{i}$ with height $\delta$ by

$$
\widetilde{W}_{i}^{\delta} \stackrel{\text { def }}{=} M_{i} \times\{\delta\} \text { and } W_{i}^{\delta} \stackrel{\text { def }}{=} \Xi\left(\widetilde{W}_{i}^{\delta}\right) .
$$

Using the coarea formula, and the fact that $W_{i} \cap \partial_{*}\left\{v_{0}>\xi\right\}=W_{i}^{0}$, for $\xi \in$ $(\alpha, \beta)$, we compute $E_{c}^{0}\left(v_{0}\right)$ as follows:

$$
\begin{aligned}
E_{c}^{0}\left(v_{0}\right) & =\int_{W_{i}} h\left|D v_{0}\right|=\int_{-\infty}^{\infty} \int_{W_{i} \cap \partial_{*}\left\{v_{0}>\xi\right\}} h d \mathcal{H}^{N-1}(x) d \xi \\
& =\int_{\alpha}^{\beta} \int_{W_{i}^{0}} h d \mathcal{H}^{N-1}(x) d \xi=(\beta-\alpha) \int_{M_{i}} \widetilde{h}(y \prime, 0) d \mathcal{H}^{N-1} .
\end{aligned}
$$

The trace of $\widetilde{v}(\cdot, \delta)$ is well defined on $\widetilde{W}_{i}^{\delta}$, for a.e. $\delta$ in $I_{\delta_{0}}$. In order to accomplish our goal we will separate the admissible functions in four distinct classes of functions in $W_{i}$. First we suppose that

(i) $\widetilde{v}=\widetilde{v}_{0}$ on $\widetilde{W}_{k}^{\delta} \cup \widetilde{W}_{k}^{-\delta}$ in the sense of traces, for some $\delta \in\left(\delta_{0} / 2, \delta_{0}\right)$.

Thus for any admissible function $v$ we have:

$$
\begin{aligned}
E_{c}^{0}(v) & \left.=\int_{W_{i}} h|D v| \geq \int_{\widetilde{W}_{i}} \widetilde{h}\left(\left(\sum_{j=1}^{N}\left|\widetilde{\mu}_{j}\right|^{2}\right)^{1 / 2}\right) \quad \text { (by Lemma }(2.4)\right) \\
& \geq \int_{\widetilde{W}_{i}} \widetilde{h}\left|\widetilde{\mu}_{N}\right| \\
& \left.\geq \int_{\widetilde{W}_{i}} \Lambda(y \prime, \delta)\left|\frac{\partial \widetilde{v}}{\partial \delta}\right| \quad \text { by the definition of } \widetilde{\mu}_{N}\right) \\
& >\int_{\widetilde{W}_{i}} \widetilde{h}(y \prime, 0)\left|\frac{\partial \widetilde{v}}{\partial \delta}\right|(\text { by }(3.3) \text { and }(\mathbf{i})) \\
& \geq(\beta-\alpha) \int_{M_{i}} \widetilde{h}(y \prime, 0) d \mathcal{H}^{N-1} \text { (by (i)) } \\
& =E_{c}^{0}\left(v_{0}\right) .
\end{aligned}
$$

Notice that the above strict inequality is possible not only by virtue of (3.3) but also because (i) implies that the total variation measure $\left|\frac{\partial \widetilde{v}}{\partial \delta}\right|$ is not identically zero. 
Now if (i) does not hold then at least one of the following cases must occur in the sense of traces of $B V$-functions:

(ii) $\widetilde{v}$ is not constant $\mathcal{H}^{N-1}$-a.e. on $\widetilde{W}_{i}^{\delta}$, for a.e. $\delta \in\left(\delta_{0} / 2, \delta_{0}\right)$.

(iii) $\widetilde{v}$ is not constant $\mathcal{H}^{N-1}$-a.e. on $\widetilde{W}_{i}^{-\delta}$, for a.e. $\delta \in\left(\delta_{0} / 2, \delta_{0}\right)$.

(iv) $\widetilde{v} \equiv \alpha, \mathcal{H}^{N-1}$-a.e. on $\widetilde{W}_{i}^{\delta}$ and $\widetilde{v} \equiv \beta, \mathcal{H}^{N-1}$-a.e. on $\widetilde{W}_{i}^{-\delta}$. Next, for $\rho_{i}>0$ (to be specified later), we define a set $\Delta \subset\left(0, \delta_{0}\right)$ by

$$
\Delta \stackrel{\text { def }}{=}\left\{\begin{array}{l}
\delta \in\left(0, \delta_{0}\right): \\
\left(\int_{\widetilde{W}_{i}^{\delta}}\left|\widetilde{v}-\widetilde{v}_{0}\right| J_{\Xi} d \mathcal{H}^{N-1}+\int_{\widetilde{W}_{i}^{-\delta}}\left|\widetilde{v}-\widetilde{v}_{0}\right| J_{\Xi} d \mathcal{H}^{N-1}\right)>\left(4 \rho_{i} / \delta_{0}\right)
\end{array}\right\}
$$

Hence $|\Delta|<(\delta / 4)$ if $\rho_{i}>\left\|v-v_{0}\right\|_{L^{1}\left(W_{i}\right)}>0$. Also we define the following positive number,

$$
J_{\Xi, \delta_{0}}=\inf _{\left(0, \delta_{0}\right)} \inf _{\widetilde{W}_{i}^{\delta}}\left\{J_{\Xi}\left(y^{\prime}, \delta\right), J_{\Xi}\left(y^{\prime},-\delta\right)\right\}
$$

If (iv) holds then by choosing

$$
\rho_{i}<\frac{1}{2}\left\{\delta_{0}(\beta-\alpha) \mathcal{H}^{N-1}\left(M_{i}\right) J_{\Xi, \delta_{0}}\right\}
$$

we obtain

$$
\begin{gathered}
\int_{\widetilde{W}_{i}^{\delta}}\left|\widetilde{v}-\widetilde{v}_{0}\right| J_{\Xi} d \mathcal{H}^{N-1}+\int_{\widetilde{W}_{i}^{-\delta}}\left|\widetilde{v}-\widetilde{v}_{0}\right| J_{\Xi} d \mathcal{H}^{N-1}= \\
2(\beta-\alpha) \mathcal{H}^{N-1}\left(M_{i}\right) J_{\Xi, \delta_{0}}>\left(4 \rho_{i} / \delta_{0}\right) .
\end{gathered}
$$

Thus if (iv) holds then necessarily $\delta \in \Delta$. Hence for a.e. $\delta \in\left(\delta_{0} / 2, \delta_{0}\right) \backslash \Delta$ either (ii) or (iii) holds. Resorting to Lemma 2.4 we obtain

$$
\begin{gathered}
E_{c}^{0}(v) \geq \int_{W_{i} \backslash\left(\cup_{\delta \in I_{\delta_{o} / 2}} W_{i}^{\delta}\right)} h|D v|+\int_{M_{i} \times I_{\delta_{0} / 2}} \widetilde{h}\left(\sum_{j=1}^{N}\left|\widetilde{\mu}_{j}\right|^{2}\right)^{1 / 2} \geq \\
\int_{W_{i} \backslash\left(\cup_{\left.\delta \in I_{\delta_{o} / 2} W_{i}^{\delta}\right)}\right.} h|D v|+\int_{M_{i} \times I_{\delta_{0} / 2}} \Lambda\left|\frac{\partial \widetilde{v}}{\partial \delta}\right|=I_{1}+I_{2}
\end{gathered}
$$

where $I_{1}$ and $I_{2}$ denote the first and second integrals respectively in the last term of the above inequalities .

Next we will be working toward finding a lower bound for $I_{i}, i=1,2$. 
Actually since $v \in B V\left(W_{i},\{\alpha, \beta\}\right)$ if (ii) holds then for a.e. $\delta \in\left(\delta_{0} / 2, \delta_{0}\right)$ the set $\widetilde{W}_{i}^{\delta} \cap \partial_{*}\{v=\alpha\} \cap \partial_{*}\{v=\beta\} \subset \mathbb{R}^{N-1}$ is rectifiable and satisfies

$$
\operatorname{Per}_{\widetilde{W}_{i}^{\delta}}\left(\partial_{*}\{v=\alpha\} \cap \partial_{*}\{v=\beta\}\right)>0 .
$$

A similar remark applies if (iii) holds. This fact will be used to estimate $I_{1}$. Further, for the sake of simplification we set in the original variables $\partial_{*}^{\alpha, \beta} \stackrel{\text { def }}{=}$ $\partial_{*}\{v=\alpha\} \cap \partial_{*}\{v=\beta\}$, and in the new variables $\widetilde{\partial}_{*}^{\alpha}, \beta \stackrel{\text { def }}{=} \partial_{*}\{\widetilde{v}=\alpha\} \cap \partial_{*}\{\widetilde{v}=\beta\}$. Also define

$$
\tau \stackrel{\text { def }}{=} \min \left\{\Lambda(y \prime, \delta), \quad(y \prime, \delta) \in \widetilde{W}_{i}, i \in\{1, \ldots, K\}\right\}
$$

and $J_{\delta_{o}, \Delta} \stackrel{\text { def }}{=}\left(\delta_{0} / 2, \delta_{0}\right) \backslash \Delta$.

Therefore

$$
\begin{aligned}
I_{1} & \geq \int_{-\infty}^{\infty} \int_{\bigcup_{\delta \in J_{\delta_{0}, \Delta}}\left(W_{i}^{\delta} \cup W_{i}^{-\delta}\right) \cap \partial_{*}\{v>\xi\}} h d \mathcal{H}^{N-1}(x) d \xi \\
& =(\beta-\alpha)\left(\int_{\bigcup_{\delta \in J_{\delta_{0}, \Delta}}\left(W_{i}^{\delta} \cup W_{i}^{-\delta}\right) \cap \partial_{*}^{\alpha, \beta}} h d \mathcal{H}^{N-1}(x)\right) \\
& =(\beta-\alpha)\left(\int_{\bigcup_{\delta \in J_{\delta_{0}, \Delta}}\left(\widetilde{W}_{i}^{\delta} \cup \widetilde{W}_{i}^{-\delta}\right) \cap \widetilde{\partial}_{*}^{\alpha, \beta}} \Lambda d \mathcal{H}^{N-1}\right) \\
& \geq \tau(\beta-\alpha)\left(\int_{\bigcup_{\delta \in J_{\delta_{o}, \Delta}}\left(\widetilde{W}_{i}^{\delta} \cup \widetilde{W}_{i}^{-\delta}\right) \cap \widetilde{\partial}_{*}^{\alpha, \beta}} d \mathcal{H}^{N-1}\right) \\
& \geq \tau(\beta-\alpha)\left(\int_{J_{\delta_{o}, \Delta}}\left(\int_{\left(\widetilde{W}_{i}^{\delta} \cup \widetilde{W}_{i}^{-\delta}\right) \cap \widetilde{\partial}_{*}^{\alpha, \beta}} d \mathcal{H}^{N-2}\right) d \delta\right)
\end{aligned}
$$

(by the coarea formula in a rectifiable set)

$$
=\tau(\beta-\alpha) \int_{J_{\delta_{0}, \Delta}} \operatorname{Per}_{\left(\widetilde{W}_{i}^{\delta} \cup \widetilde{W}_{i}^{-\delta}\right)} \widetilde{\partial}_{*}^{\alpha, \beta} d \delta .
$$

It turns out that the mapping $\delta \longrightarrow \operatorname{Per}_{\left(\widetilde{W}_{i}^{\delta} \cup \widetilde{W}_{i}^{-\delta}\right)} \widetilde{\partial}_{*}^{\alpha, \beta}$ is integrable. See [12], for instance. Setting for simplicity

$$
\mathcal{M}\left(\delta_{0}, \Delta\right) \stackrel{\text { def }}{=} \int_{J_{\delta_{0}, \Delta}} \operatorname{Per}_{\left(\widetilde{W}_{i}^{\delta} \cup \widetilde{W}_{i}^{-\delta}\right)} \widetilde{\partial}_{*}^{\alpha, \beta} d \delta
$$

we then conclude using (4.7) that $I_{1} \geq \tau(\beta-\alpha) \mathcal{M}\left(\delta_{0}, \Delta\right)>0$.

Now in order to obtain a lower estimate for $I_{2}$, note that since

$$
\left|\left(0, \delta_{0} / 2\right) \backslash \Delta\right| \geq\left(\delta_{0} / 4\right)
$$


and $\widetilde{v} \in B V\left(\widetilde{W_{i}} ;\{\alpha, \beta\}\right)$, there is $\bar{\delta} \in\left(0, \delta_{0} / 2\right) \backslash \Delta$ such that $\left(y^{\prime}, \bar{\delta}\right)$ and $(y \prime,-\bar{\delta})$ are points of approximate continuity of $\widetilde{v}$, for a.e. $y^{\prime} \in M_{i}$.

It follows from the definition of $\widetilde{v}_{0}$ that

$$
\left|\widetilde{v}\left(y^{\prime}, \delta\right)-\widetilde{v}\left(y^{\prime},-\delta\right)\right| \geq(\beta-\alpha)-\left\{\left(\left|\widetilde{v}_{0}-\widetilde{v}\right|\left(y^{\prime}, \delta\right)+\left|\widetilde{v}_{0}-\widetilde{v}\right|\left(y^{\prime},-\delta\right)\right\}\right.
$$

for any $\left(y^{\prime}, \delta\right) \in \widetilde{W}_{i}$ such that $\widetilde{v}$ is approximately continuous at $\left(y^{\prime}, \delta\right)$. In the sequel we set $h_{M} \stackrel{\text { def }}{=} \max \{h(x), x \in \bar{\Omega}\}$.

Therefore using (3.3), the above inequality and results about essential variation, it follows that

$$
\begin{aligned}
I_{2} & =\int_{M_{i} \times I_{\delta_{0} / 2}} \Lambda\left|\frac{\partial \widetilde{v}}{\partial \delta}\right| \\
& \geq \int_{M_{i}} \int_{-\delta_{0} / 2}^{\delta_{0} / 2} \widetilde{h}\left(y^{\prime}, 0\right)\left|\frac{\partial \widetilde{v}}{\partial \delta}\right|(\text { by Fubini's theorem) } \\
& =\int_{M_{i}} \widetilde{h}\left(y^{\prime}, 0\right) \text { ess } V_{-\delta_{0} / 2}^{\delta_{0} / 2}\left[\widetilde{v}\left(y^{\prime}, \cdot\right)\right] d \mathcal{H}^{N-1}\left(y^{\prime}\right) \\
\geq & \int_{M_{i}} \widetilde{h}(y \prime, 0)\left|\widetilde{v}\left(y^{\prime}, \bar{\delta}\right)-\widetilde{v}\left(y^{\prime},-\bar{\delta}\right)\right| d \mathcal{H}^{N-1}(y \prime) \\
\geq & (\beta-\alpha) \int_{M_{i}} \widetilde{h}\left(y^{\prime}, 0\right) d \mathcal{H}^{N-1}\left(y^{\prime}\right)- \\
& \left.h_{M}\left\{\int_{\widetilde{W}_{i}^{\bar{\delta}}}\left|\widetilde{v}-\widetilde{v}_{0}\right| d \mathcal{H}^{N-1}\left(y^{\prime}\right)\right)+\int_{\widetilde{W}_{i}^{-\bar{\delta}}}\left|\widetilde{v}-\widetilde{v}_{0}\right| d \mathcal{H}^{N-1}\left(y^{\prime}\right)\right\} \\
\geq & \left\{E_{c}^{0}\left(v_{0}\right)-\frac{4 \rho_{i} h_{M}}{\delta_{0} J_{\Xi, \delta_{0}}}\right\},
\end{aligned}
$$

where the fact that $\bar{\delta} \notin \Delta$ has been used. Altogether these estimates yield

$$
\begin{aligned}
E_{c}^{0}(v) & \geq I_{1}+I_{2} \\
& \geq\left\{\tau(\beta-\alpha) \mathcal{M}_{i}\left(\delta_{0}, \Delta\right)-\frac{4 \rho_{i} h_{M}}{\delta_{0} J_{\Xi, \delta_{0}}}+E_{c}^{0}\left(v_{0}\right)\right\} .
\end{aligned}
$$

Now in order to have $E_{c}^{0}(v)>E^{0}\left(v_{0}\right)$ it suffices to choose $\rho_{i}$ such that

$$
\rho_{i}<\frac{\delta_{0}}{2}(\beta-\alpha) J_{\Xi, \delta_{0}} \min \left\{\frac{\tau \mathcal{M}_{i}\left(\delta_{0}, \Delta\right)}{2 h_{M}}, \mathcal{H}^{N-1}\left(M_{i}\right)\right\}
$$

where $i \in\{1, \ldots, K\}$. 
Finally we take $v \in B V\left(N_{\delta_{0}} ;\{\alpha, \beta\}\right)$ with $0<\left\|v-v_{0}\right\|_{L^{1}\left(N_{\delta_{0}}\right)}<\rho$, where $\rho=\min \left\{\rho_{i}, i=1, \ldots, K\right\}$. Now $v_{0}$ is no longer restricted to $W_{i}$. By repeating above procedure on each $W_{j}(j=1, \ldots, K)$ with $\rho_{i}$ replaced with $\rho$ and using the property that the covering $W_{j}(j=1, \ldots, K)$ of $N_{\delta_{0}}(S)$ is disjoint along with the fact that the measure $\left|D v_{0}\right|$ is supported in $S$, we conclude that

$$
\int_{N_{\delta_{0}}(S)} h|D v|>\int_{N_{\delta_{0}}(S)} h\left|D v_{0}\right|
$$

This establishes the proof.

Lemma 4.3 There is a family $\left\{v_{\varepsilon}\right\}_{0<\varepsilon \leq \varepsilon_{0}}$ of $L^{1}$-local minimisers of $E_{c}^{\varepsilon}$ such that $v_{\varepsilon} \in C^{2, \nu}, 0<\nu \leq 1$, is a classical solution to (4.1).

Moreover $\alpha<v_{\varepsilon}(x)<\beta, \forall x \in \bar{\Omega}$ and $\left\|v_{\varepsilon}-v_{0}\right\|_{L^{1}(\Omega)} \stackrel{\varepsilon \rightarrow 0}{\longrightarrow} 0$, where $v_{0}$ is given by (3.1).

Proof: In order to apply Theorem 2.3, hypotheses (2.3.i) and (2.3.ii) must be verified. But (2.3.ii) follows from Theorem 4.1 and (2.3.i) can be proved following the same argument used in [8].

Thus the existence of the minimisers $v_{\varepsilon}, 0<\varepsilon \leq \varepsilon_{0}$, such that $\| v_{\varepsilon}-$ $v_{0} \|_{L^{1}(\Omega)} \stackrel{\varepsilon \rightarrow 0}{\longrightarrow} 0$ follows from Theorem 2.3.

Each $v_{\varepsilon}$ is a weak solution to (4.4); thus a classical solution since by a standard bootstrap argument $v_{\varepsilon} \in C^{2, \nu}, 0<\nu \leq 1$.

Now a classical argument using the maximum principle yields $\alpha<v_{\varepsilon}(x)<$ $\beta, \forall x \in \bar{\Omega}$. Hence each $v_{\varepsilon}$ is also a classical solution to (4.1), by the definition of $f_{c}$.

Note that each $v_{\varepsilon}$ is a critical point of $E_{c}^{\varepsilon}$. However in order to have $v_{\varepsilon}$ a stable stationary solution to (1.1) we should have $v_{\varepsilon}$ a local minimiser of $E^{\varepsilon}$. We state that in the next lemma whose proof is similar to the one rendered in [1] and is therefore omitted. We remark that here we need the functional $E^{\varepsilon}$ to be $C^{1}$ and that is where $\left(f_{3}\right)$ is needed. 
Lemma 4.4 The family of local minimisers $\left\{v_{\varepsilon}\right\}_{0<\varepsilon \leq \varepsilon_{0}}$ of $E_{c}^{\varepsilon}$ is also a family of local minimisers of $E^{\varepsilon}$.

Proof of Theorem 3.1 As said before we now concatenate the above results to establish the proof of Theorem 3.1 In fact (3.1.i) and (3.1.ii) have been proved in Lemma 4.3.

As for (3.1.iii), we refer to [1] where a similar case has been treated.

Thus the proof of Theorem 3.1 is established.

We now consider the case of multiple-layer pattern. Let $S_{\ell}(\ell=1, \ldots, m)$, be smooth hypersurfaces which lie inside $\Omega$ and are nested, in the sense that if $O_{\ell}$ denotes the open region enclosed by $S_{\ell}$, i.e., $S_{\ell}=\partial O_{\ell}(\ell=1, \ldots, m)$, then it holds that $O_{1} \subset O_{2} \subset \cdots \subset O_{m+1} \stackrel{\text { def }}{=} \Omega$ and $\partial O_{i} \cap \partial O_{i+1}=\emptyset(i=1, \ldots, m)$.

We set throughout

$$
\Omega_{1} \stackrel{\text { def }}{=} O_{1}, \Omega_{2}=O_{2} \backslash \bar{O}_{1}, \ldots, \Omega_{m}=O_{m} \backslash \bar{O}_{m-1}, \Omega_{m+1}=\Omega \backslash \bar{O}_{m} .
$$

For future reference we consider the following function:

$$
v_{0}=\alpha \chi_{\Omega_{\alpha}^{0}}+\beta \chi_{\Omega_{\beta}^{0}}
$$

where $\chi_{A}$ stands for the characteristic function of the set $A$ and

$$
\Omega_{\alpha}^{0} \stackrel{\text { def }}{=} \bigcup_{1 \leq j \leq m+1, j: \text { odd }} \Omega_{j} \quad, \quad \Omega_{\beta}^{0} \stackrel{\text { def }}{=} \bigcup_{1 \leq j \leq m+1, j: \text { even }} \Omega_{j}
$$

For each $S_{\ell}$ we take a disjoint covering $W_{k, \ell}(k=1, \ldots, K)$ of $N_{\delta_{0}}\left(S_{\ell}\right)$ as above and change the definitions accordingly. As in the case of just one hypersurface, let

$$
\widetilde{W}_{k, \ell} \stackrel{\text { def }}{=} \Xi_{\ell}^{-1}\left(W_{k, \ell}\right)=M_{k, \ell} \times I_{\delta_{0}}
$$

and $\widetilde{g}_{\ell}=g \circ \Xi_{\ell}$, for any function $g$ defined on $W_{k, \ell}, \widetilde{g}_{\ell}(\cdot, \delta)$ will mean that for any $y^{\prime} \in M_{k, \ell}, \widetilde{g}_{\ell}$ is considered as a function of $\delta$ alone. Note that $J_{\Xi_{\ell}}(y \prime, 0)=1$ and $\widetilde{g}_{\ell}(y \prime, 0)=g(y)$ for $y \in S_{\ell}$.

Corollary 4.1 Let $S_{\ell}(\ell=1, \ldots, m)$ be as above and suppose that (3.3) holds on each hypersurface. Then $v_{0}$, given by $(4.8)$, is a $L_{1}$-local isolated minimiser of $E_{c}^{0}$. 
Proof: Recovering the subindex that has been dropped we find positive numbers $\rho_{\ell}(\ell=1, \ldots, m)$ and $\delta_{0, \ell}(\ell=1, \ldots, m)$ whose definitions are the same as those of $\rho$ and $\delta_{0}$ in the proof of Theorem 4.2.

Define $\bar{\rho}=\min \left\{\rho_{\ell}, \ell=1, \ldots, m\right\}$ and $\bar{\delta}_{0}=\min \left\{\delta_{0, \ell}, \ell=1, \ldots, m\right\}$. Also let $v_{0, \ell}$ stand for the restriction of $v_{0}$ to $N_{\delta_{0}}\left(S_{\ell}\right)$.

Then if $v \in B V\left(N_{\bar{\delta}_{0}}\left(S_{\ell}\right) ;\{\alpha, \beta\}\right)$ with $\left\|v-v_{0, \ell}\right\|_{L^{1}\left(N_{\bar{\delta}_{0}\left(S_{\ell}\right)}\right)}<\bar{\rho}$, using Theorem 4.2 and the fact that the measure $\left|D v_{0, \ell}\right|$ is suported in $S_{\ell}$, it holds that

$$
\int_{N_{\bar{\delta}_{0}}\left(S_{\ell}\right)} h|D v|>\int_{N_{\bar{\delta}_{0}}\left(S_{\ell}\right)} h\left|D v_{0, \ell}\right| \quad \ell=1, \ldots, m .
$$

Finally take $v \in B V(\Omega ;\{\alpha, \beta\})$ such that $0<\left\|v-v_{0}\right\|_{L^{1}(\Omega)}<\bar{\rho}$. Also set $\Omega_{\alpha} \stackrel{\text { def }}{=}\{x \in \Omega: v(x)=\alpha\} \quad, \quad \Omega_{\beta} \stackrel{\text { def }}{=}\{x \in \Omega: v(x)=\beta\}$ and consider the sets $\Omega_{\alpha}^{0}$ and $\Omega_{\beta}^{0}$ defined by (4.9). Note that the set $\left(\Omega_{\alpha} \cup \Omega_{\beta}\right) \cap \Omega$ has finite perimeter and that the measure $\left|D v_{0}\right|$ is supported in $\cup_{\ell=1}^{m} S_{\ell}$. Thus keeping the notation of the previous theorem we conclude that

$$
\begin{aligned}
E_{c}^{0}(v) & =\int_{\Omega} h|D v|=(\beta-\alpha) \int_{\partial_{*} \Omega_{\alpha} \cap \partial_{*} \Omega_{\beta} \cap \Omega} h d \mathcal{H}^{N-1}(x) \\
& \geq(\beta-\alpha) \sum_{\ell=1}^{m} \int_{\partial_{*} \Omega_{\alpha} \cap \partial_{*} \Omega_{\beta} \cap N_{\delta_{0}}\left(S_{\ell}\right)} h_{\ell} d \mathcal{H}^{N-1}(x) \\
& >(\beta-\alpha) \sum_{\ell=1}^{m} \int_{\partial_{*} \Omega_{\alpha}^{0} \cap \partial_{*} \Omega_{\beta}^{0} \cap N_{\delta_{0}}\left(S_{\ell}\right)} h_{\ell} d \mathcal{H}^{N-1}(x) \text { (by (4.9)) } \\
& =(\beta-\alpha) \int_{\bigcup_{\ell=1}^{m} S_{\ell}} h_{\ell} d \mathcal{H}^{N-1}=\int_{\Omega} h\left|D v_{0}\right|=E_{c}^{0}\left(v_{0}\right) .
\end{aligned}
$$

Now we remark that since Lemmas 4.3 and 4.4 as well as Theorem 3.1 hold regardless of $v_{0}$ being a single or multiple-layer minimiser the claimed result about multiple-layer patterns follows in a analogous manner. 


\section{References}

[1] do Nascimento, A. S., Stable stationary solutions induced by spatial inhomogeneity via $\Gamma$-convergence, Bulletin of the Brazilian Mathematical Society, 29, No.1 (1998).

[2] do Nascimento, A. S., Stable multiple-layer stationary solutions of a semilinear parabolic equation in two-dimensional domains, Electronic J. Diff. Eqns., No.21 (1997), 1-17.

[3] do Nascimento, A. S., Reaction-diffusion induced stability of a spatially inhomogeneous equilibrium with boundary layer formation, J. Diff. Eqns. 108, No.2 (1994), 296-325.

[4] do Nascimento, A. S., Internal transitions layers in a elliptic boundary value problem: a necessary condition, Nonlinear Analysis: T., M. \& A. 44 (2001), 487-497.

[5] do Nascimento, A. S., Stable transitions layers in a semilinear diffusion equation with spatial inhomogeneities in $N$-dimensional domains, Journal of Differential Equations 190 (2003), 16-38.

[6] Fusco, G.; Hale, J. K., Stable equilibria in a scalar parabolic equation with variable diffusion, SIAM J. Math. Anal. 16, No.6 (1985), 1152-1164.

[7] Hale, J. K.; Sakamoto, K., Existence and stability of transition layers, Japan J. Appl. Math. 5 (1988), 367-405.

[8] Sternberg, P., The effect of a singular perturbation on nonconvex variational problems Arch. Rat. Mech. Anal., 101 (1988), 209-260.

[9] Kohn, R. V.; Sternberg, P., Local minimizers and singular perturbations Proceedings of the R. Soc. of Edinburgh, 111 A (1989), 69-84.

[10] Matano, H., Asymptotic behavior and stability of solutions of semilinear diffusion equations Publ. Res. Inst. Math. Sci., 15, No.2 (1979), 401-454. 
[11] Miyata, S.; Yanagida, E., Stable stationary solution with multiple layers in a scalar parabolic equation with variable diffusion, Funkcialaj Ekvacioj, 38 (1995), 367-380.

[12] Ziemer, W. P., Weakly Differentiable Function, Springer-Verlag (1989).

Universidade Federal de São Carlos

Departamento de Matemática

Via Washington Luiz, Km 235

13565-905 - São Carlos, S.P., Brasil
Universidade de São Paulo

Instituto de Ciências Matemáticas e

da Computação

Av. Dr. Carlos Botelho, 1465

13560-970 - São Carlos, S.P., Brasil 